\title{
Interleukin-6 as Predictor of One-Year Cognitive Function After Ischemic Stroke or TIA
}

\author{
Yu Wang ${ }^{1,2, *}$, Jiejie $\mathrm{Li}^{1,2, *}$, Yuesong Pan ${ }^{1,2}$, Mengxing Wang ${ }^{1,2}$, Jinxi Lin ${ }^{1,2}$, Xia Meng ${ }^{1,2}$, \\ Xiaoling Liao ${ }^{1,2}$, Yongjun Wang ${ }^{1-3}$ \\ 'Department of Neurology, Beijing Tiantan Hospital, Capital Medical University, Beijing, People's Republic of China; ${ }^{2}$ China National Clinical Research \\ Center for Neurological Diseases, Beijing, People's Republic of China; ${ }^{3}$ Research Unit of Artificial Intelligence in Cerebrovascular Disease, Chinese \\ Academy of Medical Sciences, Beijing, People's Republic of China
}

*These authors contributed equally to this work

Correspondence: Yongjun Wang, Department of Neurology, Beijing Tiantan Hospital, Capital Medical University, Beijing, 100070, China; China National Clinical Research Center for Neurological Diseases, Beijing, People's Republic of China, Tel +86 10-5997835I, Fax +86 I0-59978316, Email yongjunwang@ncrend.org.cn

Background and Purpose: The relationship between inflammatory markers and cognitive decline in a poststroke setting is still unclear. We aimed to investigate the association between interleukin-6 (IL-6) and cognitive decline after acute ischemic stroke and transient ischemic attack (TIA).

Methods: In this prespecified prospective substudy of the Impairment of CognitiON and Sleep after acute ischemic stroke or transient ischemic attack in Chinese patients (ICONS) study, a total of 1003 patients with baseline IL-6 levels and completed standard 3-month and 1-year cognitive function evaluation were included. Cognitive decline was defined according to a reduction of Montreal Cognitive Assessment (MoCA) $\geq 2$ between 3 months and one year. Multivariable logistic regression analysis was used to determine the association.

Results: Totally, 238 (23.73\%) patients had post-stroke cognitive decline at one year. IL-6 levels were classified into four groups according to their quartile. Patients in the highest quartile of IL-6 level had higher risk of cognitive decline than those in the first quartile $(25.90 \%$ vs $16.80 \%$, adjusted OR, 1.95 ; 95\% CI, 1.13-3.38, P = 0.0167), after adjusting for potential risk factors.

Conclusion: Elevated IL-6 levels were independently associated with reduction of Montreal Cognitive Assessment after ischemic stroke and TIA.

Keywords: inflammation, biomarker, stroke, transient ischemic attack, cognitive decline

\section{Introduction}

Stroke is a leading cause of disability worldwide. ${ }^{1}$ With over 2 million new cases annually, stroke is associated with the highest disability-adjusted life-years loss of any disease in China. ${ }^{2}$ Up to one-third of stroke patients develop post-stroke cognitive impairment (PSCI). ${ }^{3-5}$ Strokes affected daily functioning including cognition in addition to executive function, which influenced quality of life and return to work, contributing to a growing health, social and economic burden. ${ }^{6}$ However, PSCI has been an underestimated complication compared with physical disability. There is an urgent need to early screen and apply intervention treatment for the patients at high-risk of PSCI.

Inflammation has been shown to be a crucial mechanism in cognition impairment. ${ }^{7,8}$ Interleukin-6 (IL-6) was one of crucial inflammatory biomarkers. Some studies investigated the association between IL-6 and cognitive impairment in the general population; however, the conclusion was controversial, ${ }^{9-15}$ and limited information was available in stroke patients. $^{16}$

The ICONS study is a large-scale prospective national registry aiming to investigate the risk factors of cognitive impairment after ischemic stroke or transient ischemic attack (TIA). ${ }^{17}$ In this subgroup analysis, we investigated the contribution of IL-6 in one-year post-stroke cognitive impairment among patients with ischemic stroke or TIA. 


\section{Method}

\section{Study Design}

Data for this study were obtained from a prespecified prospective substudy of the ICONS study. The ICONS study was a subgroup of China National Stroke Registry-III (CNSR-III), a prospective multicenter registry that enrolled patients within seven days after symptom onset with a diagnosis of acute ischemic stroke or TIA between August 2015 and March 2018 from 201 hospitals that cover 22 provinces and 4 municipalities in China. Of these, 40 sites with experience of cognition, qualified research capability and proved commitment to the study voluntarily participated in the ICONS study. The inclusion and exclusion criteria of the ICONS study have been described before. ${ }^{17}$ The inclusion criteria of ICONS were the same as CNSR-III, including: age older than 18 years; in-hospital AIS or TIA patients within 7 days after onset. However, the following exclusion criteria were added to ICONS, including: prior diagnosis of cognitive impairment, schizophrenia or psychosis disease; illiterate patients; and concomitant Neurological disorders that interfere with cognitive or sleep evaluation, for example, severe aphasia defined as National Institutes of Health Stroke Scale (NIHSS) item 9>2, visual impairment, hearing loss, dyslexia, severe unilateral neglect or consciousness disorders. ${ }^{17}$ Acute ischemic stroke and TIA were defined according to the WHO criteria and confirmed by CT or MRI. ${ }^{18}$

Among the 40 sites in ICONS study, 28 sites voluntarily participated in the biomarker substudy, collected specimens from consecutive patients in these sites. Patients from these 28 sites with baseline IL-6 level and fulfilled 3-month and 1-year cognitive function assessments were included in this study.

This study was conducted in accordance with the Declaration of Helsinki and the protocol of CNSR-III and ICONS was approved by the ethics committee at Beijing Tiantan Hospital (IRB approval number: KY2015-001-01) and all participating centers. All patients provided written informed consent.

\section{Baseline Data Collection}

Demographics, medical history laboratory data and medication usage during hospitalization were obtained from medical records by unified trained investigators following standard protocol. ${ }^{19}$ All the Clinical data were collected through the electronic data capture system (EDC) that automatically checks for integrity and logical corrections of the uploaded data and will improve the data quality.

\section{Inflammatory Markers Assay}

Fasting blood samples were withdrawn for all patients within 24 hours of admission. Blood samples were transferred via cold chain to the central laboratory of Beijing Tiantan Hospital and measured blindly. IL-6 concentrations were measured using enzyme-linked immunosorbent assay kits (catalog number: PHS600C, R\&D Systems, Inc, Minneapolis, MN, USA).

\section{Cognitive Function Assessment}

All participants had Montreal Cognitive Assessment (MoCA) at 3-month and 1-year through face-to-face interviews with a trained research psychologist. Post-stroke cognitive decline was defined as a reduction of MoCA score $\geq 2$ between three months and one year. ${ }^{20}$

\section{Statistical Analysis}

Continuous variables were given as the median and interquartile range (IQR), whereas categorical variables were represented as frequencies and proportions. Mann-Whitney $U$-test and the Chi-square test were used for comparison between groups of baseline characteristics. IL-6 level was categorized into four groups according to their quartiles (quartile 1: $<1.59 \mathrm{ng} / \mathrm{L}$, quartile 2: 1.59 to $2.50 \mathrm{ng} / \mathrm{L}$, quartile 3: 2.50 to $4.29 \mathrm{ng} / \mathrm{L}$, quartile $4: \geq 4.29 \mathrm{ng} / \mathrm{L}$ ). We performed crude and multivariate logistic regression models to evaluate the association between IL-6 and post-stroke cognitive decline. The potential confounders were demographic factors, prior published traditional or clinical risk factors and variables associated with outcomes in univariate analysis, with a P-value of $<0.05$. 
Interactions between IL-6 and age, sex, symptomatic intracranial stenosis, or the Org 10,172 test in the Treatment of Acute Stroke (TOAST) classification and apolipoprotein E (APOE) status on the outcome were further tested by the likelihood ratio tests. In addition, we conducted a mediation analysis to determine whether stroke recurrence could mediate the effect of increased IL-6 level on the risk of cognitive decline after stroke. We used SAS 9.4 software (SAS Institute, Inc, Cary, NC) to conduct the statistical analyses. A two-sided $p$-value of less than 0.05 was considered significant.

\section{Data Availability Statement}

All anonymized data in this study could be shared by request from any qualified investigator.

\section{Results}

\section{Baseline Characteristics}

A total of 1003 consecutive patients were enrolled in this study (Figure 1). The median age was 62 (53-70) years, and 728 (72.58\%) patients were males. Among them, 238 (23.73\%) patients had post-stroke cognitive decline. Patients in the top quartile of IL-6 levels were older, likely to have history of coronary heart disease and symptomatic intracranial stenosis (Table 1). Compared with patients with no post-stroke cognitive decline, patients with post-stroke cognitive decline were older. There is no difference in other vascular risk factors between two groups (Table 2).

\section{Inflammatory Markers and Post-Stroke Cognitive Performance}

The rates of cognitive decline by quartiles of IL- 6 were $16.80 \%, 27.09 \%, 25.10 \%$, and $25.90 \%$, respectively. In crude models, higher IL-6 level was associated with increased risk of cognitive decline (quartile 2: unadjusted OR 1.84, 95\% CI 1.19-2.84; quartile 3: unadjusted OR 1.66, 95\% CI 1.07-2.57; quartile 4: unadjusted OR 1.73, 95\% CI 1.12-2.68). In the multivariate analysis, after adjusting for age, sex, education level, body mass index, current smoking, heavy drinking, medical history of stroke, transient ischemic attack, hypertension, diabetes mellitus, dyslipidemia, coronary artery disease, National Institutes of Health stroke scale score (NIHSS) at admission and symptomatic intracranial artery stenosis, significant association between IL-6 and cognitive decline were observed (quartile 2: OR 1.97, 95\% CI 1.223.18; quartile 3: OR 1.77, 95\% CI 1.09-2.87; quartile 4: OR 1.73, 95\% CI 1.05-2.85) (Figure 2). This association remained statistically significant, even further adjusting for one-year stroke recurrence, TOAST classification, white blood cell counts and APOE status (quartile 2: OR 1.94, 95\% CI 1.16-3.26; quartile 3: OR 1.73, 95\% CI 1.02-2.93; quartile 4: OR 1.95, 95\% CI 1.13-3.38) (Figure 2). The risk for the patients in the top quartile of IL-6 increased by $95 \%$

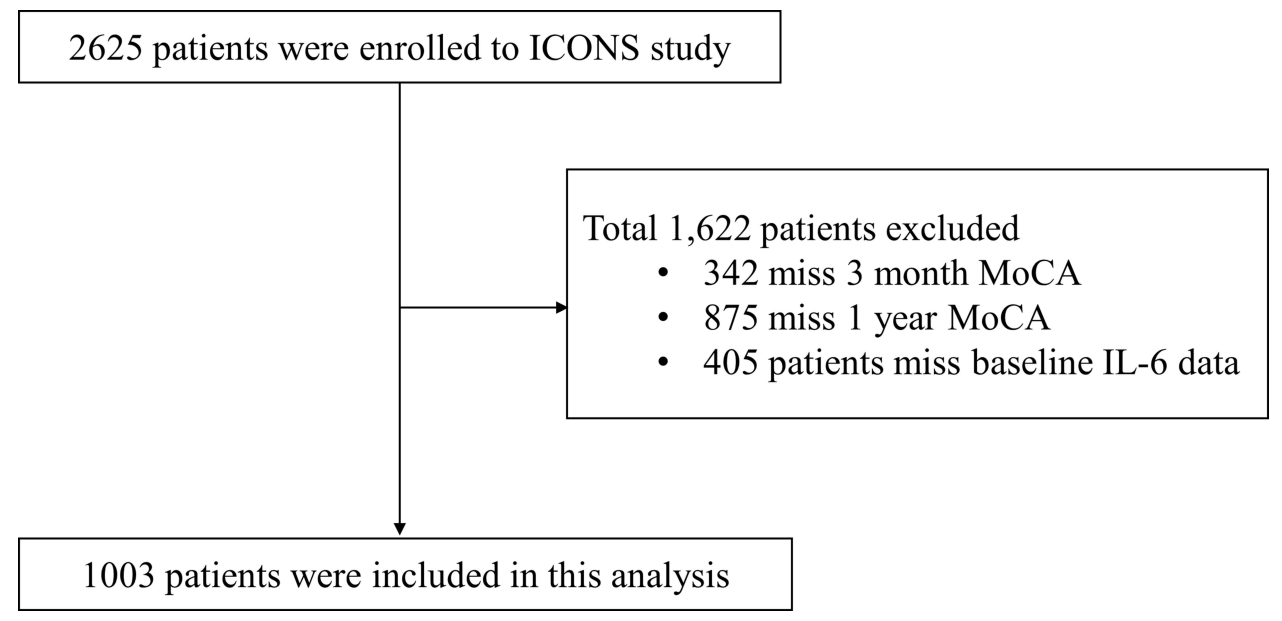

Figure I Flow chart of study population.

Abbreviations: IL-6, interleukin-6; ICONS, Impairment of CognitiON and Sleep after acute ischemic stroke or transient ischemic attack in Chinese patients; MoCA, Montreal Cognitive Assessment. 
Table I Baseline Characteristics of Participants According to IL-6 Quartiles

\begin{tabular}{|c|c|c|c|c|c|}
\hline \multirow[t]{2}{*}{ Characteristics } & \multicolumn{4}{|c|}{ IL-6 Level } & \multirow[t]{2}{*}{$P$ value } \\
\hline & $\begin{array}{c}\text { QI } \\
(<1.59)\end{array}$ & $\begin{array}{c}\text { Q2 } \\
(1.59-2.50)\end{array}$ & $\begin{array}{c}\text { Q3 } \\
(2.50-4.29)\end{array}$ & $\begin{array}{c}\text { Q4 } \\
(\geq 4.29)\end{array}$ & \\
\hline Age, year & $59(52-65)$ & $61(53-70)$ & $62(54-70)$ & $64(56-73)$ & $<0.001$ \\
\hline Male & I8I (72.40) & I82 (72.5I) & $182(72.5 I)$ & I83 (72.9I) & 0.9993 \\
\hline Education level & & & & & 0.7536 \\
\hline Elementary or below & $61(24.40)$ & $67(26.69)$ & $59(23.5 \mathrm{I})$ & $62(24.70)$ & \\
\hline Middle school & $86(34.40)$ & $78(31.08)$ & $98(39.04)$ & $93(37.05)$ & \\
\hline High school or above & $92(36.80)$ & $96(38.25)$ & $80(31.87)$ & $83(33.07)$ & \\
\hline Unknown & II (4.40) & $10(3.98)$ & $14(5.58)$ & $13(5.18)$ & \\
\hline Body mass index, $\mathrm{kg} / \mathrm{m}^{2}$ & $24.64(22.86-26.67)$ & $24.91(23.03-26.90)$ & 24.22 (22.49-26.42) & $24.49(22.60-27.04)$ & 0.0957 \\
\hline Current smoking & $89(35.60)$ & $84(33.47)$ & 91 (36.25) & $84(33.47)$ & 0.8745 \\
\hline Heavy drinking & $40(16.00)$ & $37(14.74)$ & $36(14.34)$ & $48(19.12)$ & 0.4536 \\
\hline \multicolumn{6}{|l|}{ Medical history } \\
\hline Stroke & $43(17.20)$ & $54(2 I .5 I)$ & $58(23.11)$ & $54(2 I .5 I)$ & 0.4028 \\
\hline TIA & $10(4.00)$ & $8(3.19)$ & $10(3.98)$ & $5(1.99)$ & 0.5492 \\
\hline Hypertension & $150(60.00)$ & $158(62.95)$ & $161(64.14)$ & $166(66.14)$ & 0.5457 \\
\hline Diabetes mellitus & $59(23.60)$ & $65(25.90)$ & $58(23.11)$ & $58(23.11)$ & 0.8650 \\
\hline Dyslipidemia & $25(10.00)$ & $26(10.36)$ & $35(13.94)$ & $23(9.16)$ & 0.3251 \\
\hline Coronary artery disease & $15(6.00)$ & $31(12.35)$ & $37(14.74)$ & $40(15.94)$ & 0.0033 \\
\hline Onset to enrollment time, $\mathrm{h}$ & $18.00(3.50-47.00)$ & 16.90 (4.59-39.08) & $|2.6|(2.78-4 \mid .50)$ & $10.00(3.13-37.46)$ & 0.4236 \\
\hline NIHSS at admission & $2(I-4)$ & $3(I-4)$ & $3(I-5)$ & $4(2-6)$ & $<0.001$ \\
\hline Pre stroke mRS score $2-5$ & $16(6.40)$ & II (4.38) & $10(3.98)$ & $21(8.37)$ & 0.1303 \\
\hline Index event & & & & & 0.4727 \\
\hline TIA & $25(10.00)$ & $25(9.96)$ & $26(10.36)$ & $17(6.77)$ & \\
\hline Ischemic stroke & $225(90.00)$ & $226(90.04)$ & $225(89.64)$ & $234(93.23)$ & \\
\hline Rt-PA treatment & $16(6.40)$ & 17 (6.77) & $18(7.17)$ & $23(9.16)$ & 0.6434 \\
\hline Symptomatic ICAS & $50(22.32)$ & $49(22.07)$ & $64(27.59)$ & 77 (33.77) & 0.0143 \\
\hline \multicolumn{6}{|l|}{ Medication during hospitalization } \\
\hline Antiplatelet & $246(98.8)$ & $244(98.0)$ & $248(99.2)$ & $244(97.6)$ & 0.4685 \\
\hline Anticoagulants & $17(6.8)$ & $15(6.0)$ & $10(4.0)$ & II (4.4) & 0.4475 \\
\hline Antihypertensive & $116(46.6)$ & $140(56.2)$ & $117(46.80)$ & $130(52.0)$ & 0.0945 \\
\hline Antidiabetic & $60(24.1)$ & $73(29.3)$ & $62(24.8)$ & $69(27.6)$ & 0.5162 \\
\hline Lipid-lowering agent & $245(98.4)$ & $239(96.0)$ & $242(96.8)$ & $246(98.4)$ & 0.2331 \\
\hline Statin & $24 \mid(96.8)$ & $239(96.0)$ & $24 \mid(96.4)$ & $246(98.4)$ & 0.4266 \\
\hline White blood cell count, $10^{9} / \mathrm{L}$ & $6.20(5.40-7.31)$ & $6.81(5.62-7.86)$ & $6.71(5.77-7.86)$ & $7.05(5.99-8.72)$ & $<0.001$ \\
\hline APOE $\varepsilon 4$ carriers & $31(14.0)$ & $39(18.2)$ & $47(21.1)$ & $32(15.1)$ & 0.1914 \\
\hline One-year stroke recurrence & $20(8.0)$ & $19(7.6)$ & $15(6.0)$ & $25(10.0)$ & 0.4253 \\
\hline
\end{tabular}

Note: Variables are presented as median (interquartile range) or number (\%).

Abbreviations: mRS, modified Rankin Scale; Rt-PA, recombinant tissue plasminogen activator; APOE, apolipoprotein E; TIA, transient ischemic attack; NIHSS, National Institutes of Health stroke scale; MoCA, Montreal Cognitive Assessment; ICAS, intracranial stenosis; IL-6, interleukin-6.

risk for cognitive decline. No significant mediation of stroke recurrence on the association of IL-6 with cognitive decline was found ( $\mathrm{P}>0.05$, Supplementary Table 1).

There was no significant interaction between IL-6 and age, sex, symptomatic intracranial stenosis, TOAST classification or APOE status on the risk of post-stroke cognitive decline ( $\mathrm{P}$ value for interaction $>0.05$, Figure 3 ).

\section{Discussion}

In this study, we found that IL-6 was associated with post-stroke cognitive decline at one year in patients with acute ischemic stroke or TIA. Compared to the lowest level of IL-6, the highest level of IL-6 had a 95\% increased risk of cognitive decline after stroke. 
Table 2 Baseline Characteristics of Participants According to with or without Post-Stroke Cognitive Decline

\begin{tabular}{|c|c|c|c|}
\hline Characteristics & PSNCD (n=765) & PSCD $(n=238)$ & $P$ value \\
\hline Age, year & $61(53-69)$ & $64(56-7 I)$ & 0.0041 \\
\hline Male & $558(72.94)$ & $170(7 \mid .43)$ & 0.6478 \\
\hline Education level & & & 0.1660 \\
\hline Elementary or below & $198(25.88)$ & $5 \mathrm{I}(2 \mathrm{I} .43)$ & \\
\hline Middle school & $262(34.25)$ & $93(39.08)$ & \\
\hline High school or above & $264(34.5 \mathrm{I})$ & $87(36.55)$ & \\
\hline Unknown & $4 \mid(5.36)$ & $7(2.94)$ & \\
\hline Body mass index, $\mathrm{kg} / \mathrm{m}^{2}$ & $24.61(22.77-26.73)$ & $24.80(22.86-26.67)$ & 0.9813 \\
\hline Current smoking & $273(35.69)$ & $75(31.51)$ & 0.2375 \\
\hline Heavy drinking & $123(16.08)$ & $38(15.97)$ & 0.9672 \\
\hline \multicolumn{4}{|l|}{ Medical history } \\
\hline Stroke & $149(19.48)$ & $60(25.2 I)$ & 0.0572 \\
\hline TIA & $24(3.14)$ & $9(3.78)$ & 0.6265 \\
\hline Hypertension & $483(63.14)$ & $152(63.87)$ & 0.8387 \\
\hline Diabetes mellitus & $182(23.79)$ & $58(24.37)$ & 0.8549 \\
\hline Dyslipidemia & $91(11.90)$ & $18(7.56)$ & 0.0607 \\
\hline Atrial fibrillation & $36(4.7 I)$ & $14(5.88)$ & 0.4664 \\
\hline Coronary artery disease & $90(11.76)$ & $33(13.87)$ & 0.3882 \\
\hline Onset to enrollment time, $\mathrm{h}$ & $15.55(3.32-44.50)$ & $10.77(2.92-31.35)$ & 0.1477 \\
\hline NIHSS at admission & $3.00(1.00-5.00)$ & $3.00(1.00-5.00)$ & 0.3631 \\
\hline Pre stroke mRS score 2-5 & $47(6.14)$ & II (4.62) & 0.3797 \\
\hline Index event & & & 0.1947 \\
\hline TIA & $76(9.93)$ & $17(7.14)$ & \\
\hline Ischemic stroke & $689(90.07)$ & 221 (92.86) & \\
\hline Rt-PA treatment & 55 (7.19) & $19(7.98)$ & 0.6825 \\
\hline Symptomatic ICAS & I9I (27.44) & $49(23.33)$ & 0.2369 \\
\hline \multicolumn{4}{|l|}{ Medication during hospitalization } \\
\hline Antiplatelet & $748(98.3)$ & $234(98.7)$ & 0.6358 \\
\hline Anticoagulants & $45(5.9)$ & $8(3.4)$ & 0.1282 \\
\hline Antihypertensive & $379(49.8)$ & $124(52.3)$ & 0.4984 \\
\hline Antidiabetic & $196(25.8)$ & $68(28.7)$ & 0.3708 \\
\hline Lipid-lowering agent & $74 \mid(97.4)$ & $231(97.5)$ & 0.9351 \\
\hline Statin & $738(97.0)$ & $229(96.6)$ & 0.9768 \\
\hline White blood cell count, $10^{9} / \mathrm{L}$ & $6.70(5.59-8.00)$ & $6.54(5.70-7.82)$ & 0.6515 \\
\hline APOE $\varepsilon 4$ carriers & $113(17.2)$ & $36(17.0)$ & 0.9485 \\
\hline One-year stroke recurrence & $55(7.2)$ & $24(10.1)$ & 0.1477 \\
\hline
\end{tabular}

Note: Variables are presented as median (interquartile range) or number (\%).

Abbreviations: mRS, modified Rankin Scale; Rt-PA, recombinant tissue plasminogen activator; APOE, apolipoprotein E; PSCD, post-stroke cognitive decline; PSNCI, post-stroke no cognitive decline; TIA, transient ischemic attack; NIHSS, National Institutes of Health stroke scale; MoCA, Montreal Cognitive Assessment; ICAS, intracranial stenosis.

Several studies have investigated the correlation between IL-6 and cognitive impairment, but the conclusion is controversial. ${ }^{9-14}$ The Women's Health and Aging Study II provided evidence that higher IL-6 level was associated with greater declines over 9 years in community-dwelling older women. ${ }^{14}$ Singh-Manoux et al found that midlife IL-6 predicted cognitive decline, particularly in the reasoning domain. ${ }^{10}$ In addition, the Prospective Study with Pravastatin in the Elderly at Risk (PROSPER) study demonstrated the IL-6-174 CC genotype was associated with worse cognitive performance, provided the preliminary genetic evidence for the potential causal association. ${ }^{9}$ However, several studies demonstrated negative association. ${ }^{11,12}$ In a longitudinal study of older adults from Amsterdam, researchers found declines in processing speed over a 3-year follow-up, but only for high levels of $\alpha 1$-antichymotrypsin, not for high IL-6 levels. ${ }^{11}$ One of the reasons for this discrepancy might be the difference in study design and populations. On the other hand, much of the prior evidence is based on the general population, the association between IL-6 and cognitive 


\begin{tabular}{|c|c|c|c|c|c|c|c|c|c|c|}
\hline \multirow{2}{*}{\multicolumn{2}{|c|}{ Events (\%) }} & \multicolumn{2}{|l|}{ Model 1} & \multicolumn{2}{|l|}{ Model 2} & \multicolumn{2}{|l|}{ Model 3} & \multicolumn{2}{|l|}{ Model 4} & \\
\hline & & OR $(95 \% \mathrm{Cl})$ & P Value & OR $(95 \% \mathrm{Cl})$ & $P$ Value & OR $(95 \% \mathrm{Cl})$ & P Value & OR $(95 \% \mathrm{Cl})$ & P Value & \\
\hline Q1 & $42(16.80)$ & Reference & - & Reference & - & Reference & - & Reference & - & \\
\hline Q2 & $68(27.09)$ & $1.76(1.14-2.72)$ & 0.011 & $1.97(1.22-3.18)$ & 0.006 & $1.91(1.18-3.10)$ & 0.008 & $1.94(1.16-3.26)$ & 0.012 & 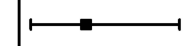 \\
\hline Q3 & $63(25.10)$ & $1.57(1.01-2.43)$ & 0.047 & $1.77(1.09-2.87)$ & 0.022 & $1.75(1.07-2.86)$ & 0.025 & $1.73(1.02-2.93)$ & 0.041 & $\longrightarrow$ \\
\hline Q4 & $65(25.90)$ & $1.56(1.00-2.44)$ & 0.050 & $1.73(1.05-2.85)$ & 0.031 & $1.74(1.05-2.89)$ & 0.033 & $1.95(1.13-3.38)$ & 0.017 & $\longmapsto$ \\
\hline
\end{tabular}

Figure 2 Association between levels of IL-6 and I-year post-stroke cognitive decline. Patients were categorized into 4 groups according to quartiles of IL-6 levels. Logistic regression models were used to evaluate the association between IL-6 and post-stroke cognitive decline defined as a reduction of Montreal Cognitive Assessment (MoCA) $\geq 2$ between 3 months and one year. Model I: adjusted age, sex; Model 2: adjusted for age, sex, education level, body mass index, current smoking, heavy drinking, medical history of stroke, transient ischemic attack, hypertension, diabetes mellitus, dyslipidemia, coronary artery disease, National Institutes of Health stroke scale score at admission and symptomatic intracranial artery stenosis; Model 3: adjusted for model 2 and I-year stroke recurrence, TOAST classification and white blood cell counts; Model 4: adjusted for model 3 and APOE status.

Abbreviations: APOE, apolipoprotein E; IL-6, interleukin-6; OR, odds ratio; Cl, confidence intervals; TOAST, Trial of Org I0I72 in Acute Stroke Treatment.

\section{No. of outcomes/patients (\%)}

\section{Low IL-6 level}

Age, years

$$
\leq 65
$$

$>65$

Sex

male

female

ICAS

Yes

No

\section{TOAST classification}

Large-artery atherosclerosis

Cardioembolism

Small-artery occlusion

Other

Unknown

\section{APOE status}

$\varepsilon 4$ non-carriers

$\varepsilon 4$ carriers

$68(19.60)$
$42(27.27)$
$76(20.94)$
$34(24.64)$
$74(21.33)$
$21(21.21)$

22(20.75)

$7(28.00)$

28(21.21)

$52(22.51)$

87(23.58)

13(18.06)
High IL-6 level

OR $(95 \% \mathrm{Cl}) \quad \mathrm{P}$ value Pinteration

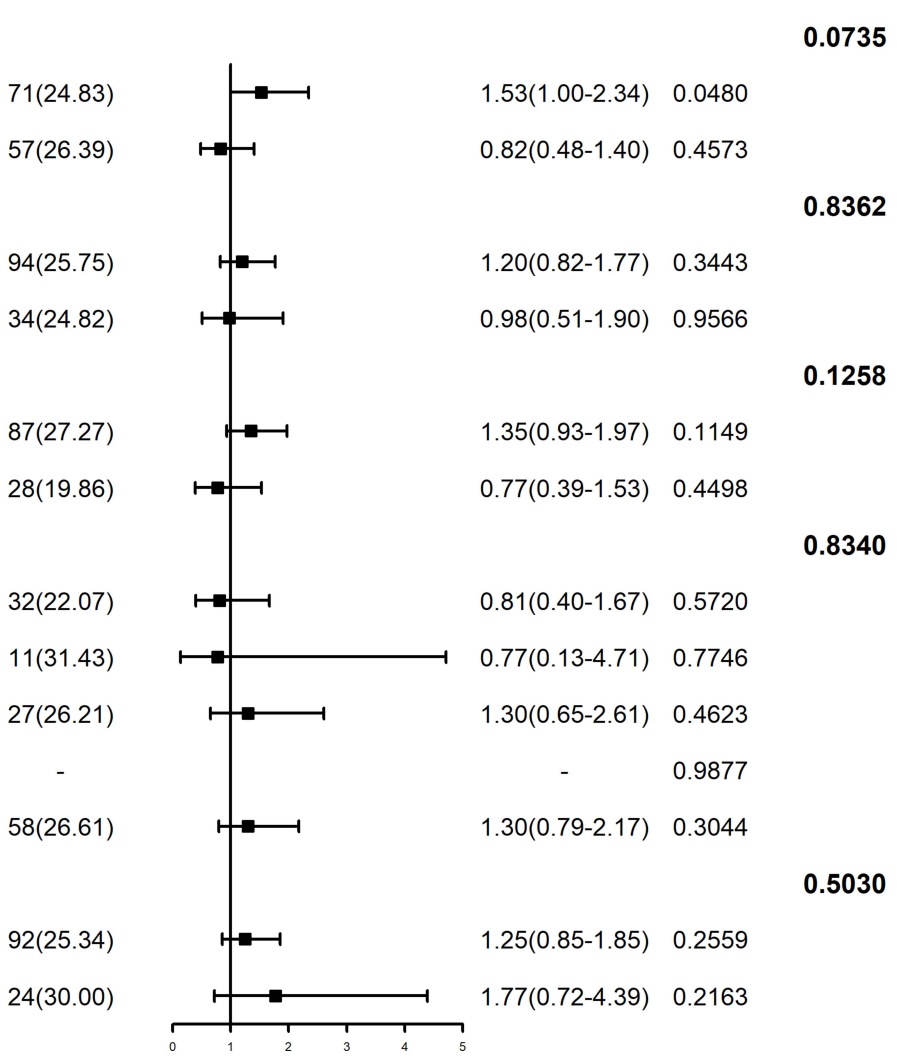

Figure 3 Subgroup analyses of the association between IL-6 level and post-stroke cognitive decline. In the multivariate models, confounding factors, such as age, sex, education level, body mass index, current smoking, heavy drinking, medical history of stroke, transient ischemic attack, hypertension, diabetes mellitus, dyslipidemia, coronary artery disease, National Institutes of Health stroke scale score at admission, symptomatic intracranial stenosis and one-year stroke recurrence were included unless the variables were used as a subgroup study.

Abbreviations: APOE, apolipoprotein E; IL-6, interleukin-6; OR, odds ratio; Cl, confidence intervals; ICAS, intracranial stenosis; TOAST, Trial of Org I0I72 in Acute Stroke Treatment.

decline, especially in stroke patients, however, is not yet established. ${ }^{9,16}$ A prior small sample-sized study found that IL-6 correlated with MMSE scores; however, such relationship disappeared after adjusting for other risk factors. ${ }^{21}$ Cognitive function was influenced by many elements, such as age, education level and the state of cerebral blood flow, indicating that discordance of included confounding factors might influence the predictive value of IL-6. Therefore, full adjustment for pertinent predictors is necessary. Previous studies have demonstrated that recurrent stroke during 
follow-up increased the risk of new dementia. ${ }^{22}$ The risk of post-stroke dementia after recurrent stroke is at least 2-fold higher than after the first stroke, more than a third of patients had dementia after recurrent stroke. ${ }^{23}$ We therefore further adjusted stroke recurrence in the current study and explore to what extent, if any, the relationship between IL-6 and cognitive decline was explained by recurrent stroke. However, we did not find any effect from recurrence, suggesting that the association between IL- 6 and cognitive decline could not be fully explained by stroke recurrence. In addition, we adjusted for NIHSS scores as they may affect both cognitive function and levels of inflammatory markers.

The pathway IL-6 leading to post-stroke cognition is currently unclear. The chronic inflammatory response after stroke may trigger neurotoxic pathways causing progressive degeneration. Damaged neurons may also exacerbate neuroinflammation-mediated disorders by producing chemokines and activating microglia and astrocytes. ${ }^{24}$ Strokeinduced hypoperfusion can contribute to oxidative stress and endothelial damage, which in turn promote or accelerate neuroinflammation, disruption of the blood-brain barrier, and neurodegeneration. ${ }^{25}$ Another assumption was that systemically produced IL-6 might cross the blood-brain barrier and cause damage in the brain. ${ }^{26,27}$ Experimental evidence supporting this hypothesis derived from the fact that mice overexpressing pro-inflammatory cytokines in the central nervous system exhibit neurodegeneration and cognitive decline. ${ }^{28}$ Taken together, other targeted therapy for prevention of cognitive decline might be needed for the patients with ischemic stroke in addition to existing standard secondary prevention.

In the current study, we used MoCA and evaluated cognitive change between 1-year and three months. Several studies have previously used the Neuropsychological Test Battery and the Mini-Mental State Examination to evaluate cognitive abilities. ${ }^{29-32}$ The major drawback of the long-duration Neuropsychological Test Battery is its lack of feasibility in routine practice or large-scale research. Limitations of the Mini-Mental State Examination are its ceiling effect and lack of assessment of executive defects. ${ }^{33}$ Consequently, MoCA was currently recommended to screen for post-stroke cognitive impairment and is considered the most sensitive screening tool. ${ }^{33-36}$ Furthermore, Wong et al proposed that conventional single cut-off scores were associated with a high rate of misclassification, especially in older and less-educated stroke patients. ${ }^{37}$

Overall, our results are useful in identifying patients at higher risk of developing post-stroke cognitive decline. The effect of lowering IL-6 levels in preventing or delaying post-stroke cognitive decline and related mechanisms need to be verified in further clinical trials.

Our study had several limitations. First, only one time-point maker measurement is available, which precluded us from assessing the contribution of changes in these markers over time. Second, though MoCA was a useful and practical tool to evaluate cognition, it could not provide information regarding the domain-specific function of cognition. Further investigation with a standardized battery of cognitive tests allowed detailed analyses of cognitive decline were warranted.

\section{Conclusions}

Elevated IL-6 level was associated with increased risk of reduction of Montreal Cognitive Assessment in patients with ischemic stroke or TIA. This study provides evidence that inflammation may be involved in the underlying mechanism of post-stroke cognitive decline.

\section{Data Sharing Statement}

The data that support the findings of this study are available from the corresponding author upon reasonable request.

\section{Ethical Statement}

The authors are accountable for all aspects of the work in ensuring that questions related to the accuracy or integrity of any part of the work are appropriately investigated and resolved. The CNSR-III was approved by the ethics committee at Beijing Tiantan Hospital (IRB approval number: KY2015-001-01) and all participating centers. All patients have provided written informed consent. 


\section{Acknowledgments}

We appreciated all the study participants and their relatives for supporting the CNSR-III and the ICONS study.

\section{Author Contributions}

All authors made substantial contributions to conception and design, acquisition of data, or analysis and interpretation of data; took part in drafting the article or revising it critically for important intellectual content; agreed to submit to the current journal; gave final approval of the version to be published; and agree to be accountable for all aspects of the work.

\section{Funding}

This work was supported by grants from the Capital's Funds for Health Improvement and Research (2020-1-2041), grants from Chinese Academy of Medical Sciences Innovation Fund for Medical Sciences (2019-I2M-5-029), grant from National Key R\&D Program of China (2018YFC1312903), grants from the National Natural Science Foundation of China (81870905, U20A20358), grants from Beijing Municipal Science \& Technology Commission (D171100003017002, Z181100001818001).

\section{Disclosure}

The authors report no conflicts of interest in this work.

\section{References}

1. Global, regional, and national burden of stroke. 1990-2016: a systematic analysis for the Global Burden of Disease Study 2016. Lancet Neurol. 2019;18(5):439-458.

2. Wu S, Wu B, Liu M, et al. Stroke in China: advances and challenges in epidemiology, prevention, and management. Lancet Neurol. 2019;18 (4):394-405.

3. Cumming TB, Marshall RS, Lazar RM. Stroke, cognitive deficits, and rehabilitation: still an incomplete picture. Int J Stroke. 2013;8(1):38-45.

4. Cumming TB, Brodtmann A, Darby D, Bernhardt J. The importance of cognition to quality of life after stroke. J Psychosom Res. 2014;77 (5):374-379.

5. Gorelick PB, Nyenhuis D. Stroke and cognitive decline. JAMA. 2015;314(1):29-30.

6. Fride Y, Adamit T, Maeir A, et al. What are the correlates of cognition and participation to return to work after first ever mild stroke? Top Stroke Rehabil. 2015;22(5):317-325.

7. Tangestani Fard M, Stough C, Review A. Hypothesized model of the mechanisms that underpin the relationship between inflammation and cognition in the elderly. Front Aging Neurosci. 2019;11:56.

8. Gorelick PB. Role of inflammation in cognitive impairment: results of observational epidemiological studies and clinical trials. Ann N Y Acad Sci. 2010;1207:155-162.

9. Mooijaart SP, Sattar N, Trompet S, et al. Circulating interleukin-6 concentration and cognitive decline in old age: the PROSPER study. J Intern Med. 2013;274(1):77-85.

10. Singh-Manoux A, Dugravot A, Brunner E, et al. Interleukin-6 and C-reactive protein as predictors of cognitive decline in late midlife. Neurology. 2014;83(6):486-493.

11. Dik MG, Jonker C, Hack CE, Smit JH, Comijs HC, Eikelenboom P. Serum inflammatory proteins and cognitive decline in older persons. Neurology. 2005;64(8):1371-1377.

12. Teunissen CE, van Boxtel MP, Bosma $\mathrm{H}$, et al. Inflammation markers in relation to cognition in a healthy aging population. J Neuroimmunol. 2003;134(1-2):142-150.

13. Weaver JD, Huang MH, Albert M, Harris T, Rowe JW, Seeman TE. Interleukin-6 and risk of cognitive decline: macArthur studies of successful aging. Neurology. 2002;59(3):371-378.

14. Palta P, Xue QL, Deal JA, Fried LP, Walston JD, Carlson MC. Interleukin-6 and C-reactive protein levels and 9-year cognitive decline in community-dwelling older women: the women's health and aging Study II. J Gerontol a Biol Sci Med Sci. 2015;70(7):873-878.

15. Schram MT, Euser SM, de Craen AJ, et al. Systemic markers of inflammation and cognitive decline in old age. J Am Geriatr Soc. 2007;55 (5):708-716.

16. Narasimhalu K, Lee J, Leong YL, et al. Inflammatory markers and their association with post stroke cognitive decline. Int $j$ Stroke. 2015;10 (4):513-518.

17. Wang Y, Liao X, Wang C, et al. Impairment of cognition and sleep after acute ischaemic stroke or transient ischaemic attack in Chinese patients: design, rationale and baseline patient characteristics of a nationwide multicentre prospective registry. Stroke Vasc Neurol. 2020; 6(1):svn-2020000359 .

18. Stroke-1989. Recommendations on stroke prevention, diagnosis, and therapy. Report of the WHO task force on stroke and other cerebrovascular disorders. Stroke. 1989;20(10):1407-1431.

19. Wang Y, Jing J, Meng X, et al. The Third China National Stroke Registry (CNSR-III) for patients with acute ischaemic stroke or transient ischaemic attack: design, rationale and baseline patient characteristics. Stroke Vasc Neurol. 2019;4(3):158-164.

20. Krishnan K, Rossetti H, Hynan LS, et al. Changes in Montreal Cognitive Assessment scores over time. Assessment. 2017;24(6):772-777. 
21. Rothenburg LS, Herrmann N, Swardfager W, et al. The relationship between inflammatory markers and post stroke cognitive impairment. $J$ Geriatr Psychiatry Neurol. 2010;23(3):199-205.

22. Pendlebury ST, Rothwell PM, Oxford Vascular S. Incidence and prevalence of dementia associated with transient ischaemic attack and stroke: analysis of the population-based Oxford Vascular Study. Lancet Neurol. 2019;18(3):248-258.

23. Pendlebury ST, Rothwell PM. Prevalence, incidence, and factors associated with pre-stroke and post-stroke dementia: a systematic review and meta-analysis. Lancet Neurol. 2009;8(11):1006-1018.

24. Zhang L, Yang L. Anti-inflammatory effects of vinpocetine in atherosclerosis and ischemic stroke: a review of the literature. Molecules. 2014;20 (1):335-347.

25. Jin R, Yang G, Li G. Inflammatory mechanisms in ischemic stroke: role of inflammatory cells. J Leukoc Biol. 2010;87(5):779-789.

26. Banks WA, Kastin AJ, Broadwell RD. Passage of cytokines across the blood-brain barrier. Neuroimmunomodulation. 1995;2(4):241-248.

27. Barrientos RM, Frank MG, Watkins LR, Maier SF. Memory impairments in healthy aging: role of aging-induced microglial sensitization. Aging Dis. 2010;1(3):212-231.

28. Campbell IL, Stalder AK, Chiang CS, et al. Transgenic models to assess the pathogenic actions of cytokines in the central nervous system. Mol Psychiatry. 1997;2(2):125-129.

29. Ballard C, Rowan E, Stephens S, Kalaria R, Kenny RAJS. Prospective follow-up study between 3 and 15 months after stroke: improvements and decline in cognitive function among dementia-free stroke survivors> 75 years of age. Stroke. 2003;34(10):2440-2444.

30. Douiri A, Rudd AG, Wolfe CDJS. Prevalence of poststroke cognitive impairment: South London stroke register 1995-2010. Stroke. 2013;44 (1): $138-145$.

31. Hochstenbach JB, den Otter R, Mulder TW. Cognitive recovery after stroke: a 2-year follow-up. Arch Phys Med Rehabil. 2003;84(10):1499-1504.

32. Patel M, Coshall C, Rudd AG, Wolfe CD. Natural history of cognitive impairment after stroke and factors associated with its recovery. Clin Rehabil. 2003;17(2):158-166.

33. Tombaugh TN, McIntyre NJ. The mini-mental state examination: a comprehensive review. J Am Geriatr Soc. 1992;40(9):922-935.

34. Stolwyk RJ, O’Neill MH, McKay AJ, Wong DK. Are cognitive screening tools sensitive and specific enough for use after stroke? A systematic literature review. Stroke. 2014;45(10):3129-3134.

35. Lees R, Selvarajah J, Fenton C, et al. Test accuracy of cognitive screening tests for diagnosis of dementia and multidomain cognitive impairment in stroke. Stroke. 2014;45(10):3008-3018.

36. Hachinski V, Iadecola C, Petersen RC, et al. National Institute of Neurological Disorders and Stroke-Canadian stroke network vascular cognitive impairment harmonization standards. Stroke. 2006;37(9):2220-2241.

37. Wong A, Law LS, Liu W, et al. Montreal cognitive assessment: one cutoff never fits all. Stroke. 2015;46(12):3547-3550.

Neuropsychiatric Disease and Treatment

Dovepress

\section{Publish your work in this journal}

Neuropsychiatric Disease and Treatment is an international, peer-reviewed journal of clinical therapeutics and pharmacology focusing on concise rapid reporting of clinical or pre-clinical studies on a range of neuropsychiatric and neurological disorders. This journal is indexed on PubMed Central, the 'PsycINFO' database and CAS, and is the official journal of The International Neuropsychiatric Association (INA). The manuscript management system is completely online and includes a very quick and fair peer-review system, which is all easy to use. Visit http://www.dovepress.com/testimonials.php to read real quotes from published authors.

Submit your manuscript here: https://www.dovepress.com/neuropsychiatric-disease-and-treatment-journal 